Open Access

\title{
The respiratory pressure-abdominal volume curve in a porcine model
}

\author{
Adrian Regli ${ }^{1,2,3,4^{*}}$ (D), Bart Leon De Keulenaer ${ }^{1,3}$, Bhajan Singh ${ }^{6,7,8}$, Lisen Emma Hockings ${ }^{2,5}$, Bill Noffsinger ${ }^{6,8}$ \\ and Peter Vernon van Heerden ${ }^{2,3,9}$
}

\author{
* Correspondence: \\ adrian.regli@gmail.com; \\ adrian.regli@health.wa.gov.au \\ In part abstracts have been \\ presented at the German \\ Anesthesia Congress (DAC) in \\ Leipzig 2016 and at the Annual \\ Scientific Meeting of the Australian \\ and New Zealand Intensive Care \\ Society in Perth 2016. \\ ${ }^{1}$ Intensive Care Unit, Fiona Stanley \\ Hospital, 102-118 Murdoch Drive, \\ Murdoch (Perth), WA 6150, Australia \\ ${ }^{2}$ Intensive Care Unit, Sir Charles \\ Gairdner Hospital, Hospital Avenue, \\ Nedlands (Perth), WA 6009, Australia \\ Full list of author information is \\ available at the end of the article
}

\begin{abstract}
Background: Increasing intra-abdominal volume (IAV) can lead to intra-abdominal hypertension (IAH) or abdominal compartment syndrome. Both are associated with raised morbidity and mortality. IAH can increase airway pressures and impair ventilation. The relationship between increasing IAV and airway pressures is not known. We therefore assessed the effect of increasing IAV on airway and intra-abdominal pressures (IAP).

Methods: Seven pigs $(41.4+/-8.5 \mathrm{~kg}$ ) received standardized anesthesia and mechanical ventilation. A latex balloon inserted in the peritoneal cavity was inflated in 1-L increments until IAP exceeded $40 \mathrm{cmH}_{2} \mathrm{O}$. Peak airway pressure $\left(\mathrm{pP}_{\text {Aw }}\right)$, respiratory compliance, and IAP (bladder pressure) were measured. Abdominal compliance was calculated. Different equations were tested that best described the measured pressure-volume curves.

Results: An exponential equation best described the measured pressure-volume curves. Raising IAV increased $\mathrm{PP}_{\mathrm{AW}}$ and IAP in an exponential manner. Increases in IAP were associated with parallel increases in $\mathrm{PP}_{\mathrm{AW}}$ with an approximate $40 \%$ transmission of IAP to $\mathrm{PP}_{\mathrm{AW}}$. The higher the IAP, the greater IAV effected $\mathrm{PP}_{\mathrm{AW}}$ and IAP.

Conclusions: The exponential nature of the effect of IAV on $\mathrm{PP}_{\mathrm{AW}}$ and IAP implies that, in the presence of high grades of IAH, small reductions in IAV can lead to significant reductions in airway and abdominal pressures. Conversely, in the presence of normal IAP levels, large increases in IAV may not affect airway and abdominal pressures.
\end{abstract}

Keywords: Intra-abdominal pressure, Intra-abdominal hypertension, Abdominal compliance, Airway pressures, Respiratory compliance, Abdominal volume

\section{Background}

Intra-abdominal hypertension (IAH) is defined as a sustained intra-abdominal pressure (IAP) $\geq 12 \mathrm{mmHg}$ [1]. IAH is common in critically ill patients [2] and is associated with an increased morbidity and mortality [3]. IAH is caused by additional intraabdominal volume (IAV) within the confined abdominal cavity (e.g., retroperitoneal bleed, free fluid from massive fluid resuscitation, ascites, ileus with dilated bowel, etc.) or by reduced compliance of the abdominal wall (e.g., obesity or eschars in burns patients). If IAP is significantly increased or persists, an abdominal compartment syndrome may develop which is defined as a sustained IAP $>20 \mathrm{mmHg}$ that is

(c) The Author(s). 2017 Open Access This article is distributed under the terms of the Creative Commons Attribution 4.0 International License (http://creativecommons.org/licenses/by/4.0/), which permits unrestricted use, distribution, and reproduction in any medium, provided you give appropriate credit to the original author(s) and the source, provide a link to the Creative Commons license, and indicate if changes were made. 
associated with new onset organ dysfunction [1]. Organ failure may include cardiac, respiratory, renal, and/or gastro-intestinal failure.

It has long been thought that a linear relationship exists between IAV and IAP [4-6]. However, in a recent review article, by extracting all available human IAV and IAP measurements from current literature, we were able to demonstrate an exponential relationship between IAV and IAP [7]. The exponential relationship between IAV and IAP is of interest because of the clinical consequences of IAH. Patients with IAH often have impaired lung function due to a cephaled displacement of the diaphragm, associated with impaired lung volumes and increased airway pressures resulting in difficulties in maintaining adequate ventilation [8].

Several therapeutic options exist to reduce IAP $[9,10]$. These therapies are associated with small reductions in IAV and are therefore thought to have a small effect on IAP. However, due to the exponential relationship at higher IAP ranges, small reductions in IAV may indeed have significant effects on IAP.

The effect of changes in IAV on airway pressures is well known. We therefore aimed to characterize the influence of IAV on both IAP and airway pressures.

\section{Methods}

Seven pigs were studied in a protocol to measure IAP and airway pressures caused by incremental increases in IAV. The Animal Ethics Committee of the University of Western Australia approved the study protocol (UWA RA/3/100/688). The study conformed to the regulations of the Australian code of practice for the care and use of animals for scientific purposes. Anesthesia, mechanical ventilation, surgical preparation, and instrumentation were performed as previously described [11] and are briefly outlined below.

\section{Animals}

Seven Large White breed pigs [mean (SD) animal weight of $41.4(+/-8.5) \mathrm{kg}$ ] received standardized anesthesia including initial sedation using intramuscular zolazepam/tiletamine (Zoletil ${ }^{\circ}$ ) and xylazine followed by a combination of propofol, morphine, and ketamine for maintenance of anesthesia. At the end of the experimental protocol the pigs were euthanized with intravenous pentobarbitone. No neuromuscular blocking agents were used as they are infrequently used in our clinical practice and also to reduce the risk of awareness of pain in the animals. Adequacy of the depth of anesthesia was regularly assessed (lack of muscle tone, absence of spontaneous ventilatory effort).

\section{Mechanical ventilation and airway parameters}

Mechanical ventilation (Servo 900, Siemens, Berlin, Germany) was maintained using constant tidal volumes of $8 \mathrm{~mL} / \mathrm{kg}$. Initial PEEP was $5 \mathrm{cmH}_{2} 0$. Respiratory rate was adjusted to maintain an end-tidal $\mathrm{CO}_{2}$ between 35 and $45 \mathrm{mmHg}$ before the abdomen was inflated but not changed thereafter. Peak inspiratory pressure $\left(\mathrm{pP}_{\mathrm{AW}}\right)$ and dynamic respiratory system compliance $\left(\mathrm{C}_{\mathrm{RS}}\right)$ were obtained automatically from the ventilator. End-expiratory lung volume was measured at baseline IAP and PEEP of $5 \mathrm{cmH}_{2} \mathrm{O}$ using the multiple breath nitrogen wash-out method as previously described [11]. Pressure-volume (P-V) curves were performed at PEEP $5 \mathrm{cmH}_{2} \mathrm{O}$ and then at PEEP $15 \mathrm{cmH}_{2} \mathrm{O}$. 
Intra-abdominal pressure measurement

Urinary bladder pressure was used to assess IAP. For this, a $12 \mathrm{~F}$ Foley catheter was placed in the urinary bladder via a caudal midline laparotomy and attached to a standard transducer system (Hospira, Lake Forest, IL). Mean pressures were measured from the mid-axillary line. Throughout the study, the animals remained in the supine position. A standardized injection volume of $25 \mathrm{~mL}$ of $0.9 \% \mathrm{NaCl}$ (AbViser 300, Wolfe Tory Medical, Salt Lake City, UT) was used and $60 \mathrm{~s}$ relaxation time was allowed for before definitive measurement [1]. IAH was graded as recommended by the World Society of Abdominal Compartment Syndrome [1].

\section{Abdominal pressure-volume curve}

A large intra-abdominal balloon (200 g latex weather balloon, Scientific Sales, Lawrenceville, $\mathrm{NJ}$ ) was placed in the peritoneal cavity via midline laparotomy. Even placement of the balloon in the abdomen was ensured by visual inspection and partial inflation. A 1-L precision syringe (Vitalograph, Hamburg, Germany) was used to add air to the IAV in 1-L incremental steps. After each addition to IAV, we waited $10 \mathrm{~s}$ to allow pressures to equilibrate before assessing all parameters. Abdominal inflation was not continued beyond an IAP of $40.8 \mathrm{cmH}_{2} \mathrm{O}(30.0 \mathrm{mmHg})$.

\section{Analysis and statistics}

Absolute abdominal pressure-volume points were entered in a spreadsheet and analyzed using Excel (Microsoft, Redmond, WA, USA). All pressures were converted from millimeter of mercury into centimeter of water for better comparison between the IAP and $\mathrm{pP}_{\mathrm{AW}}\left(1 \mathrm{mmHg}=1.3595 \mathrm{cmH}_{2} \mathrm{O}\right)$. The change in IAV following addition of air to the intra-abdominal balloon was pressure-corrected using the Boyle equation (pressure-corrected additional IAV $=$ measured additional $\times 1033 /(1033+$ IAP in centimeter of water), to compensate for the compressibility of air.

Two different equations were assessed for their accuracy at describing the IAPIAV curve. First, the Venegas equation, $V=a+\left[b /\left(1+e^{-(P-c) / d}\right)\right]$, a logistic function was used. This has been used to describe the characteristic sigmoid shape of pulmonary [12] and other P-V curves [13]. In the original paper, $V$ represents inflation or absolute lung volume, $P$ represents airway opening or transpulmonary pressure, and $a, b, c$, and $d$ represent fitting parameters. In the tested situation, $V$ represented additional IAV, $P$ represented absolute pressure (IAP or pPAW), and $a, b, c$, and $d$ represent fitting parameters.

Second, an alternate exponential equation, $V=v+k \times \operatorname{Ln}\left(P_{0}-p\right)$ where $V$ represented additional IAV, $P_{0}$ represented resting IAP (no additional IAV), and $v, k, p$ represent fitting parameters, was tested. This equation has been used in the past to characterize lung elastic recoil and in other instances where pressures rise in near asymptotic fashion [14]. This exponential equation is single ended and exhibits near asymptotic shape at high volumes only whereas the Venegas equation exhibits true asymptotic shape at high and low volumes.

For each corresponding P-V data set, we used the Excel "Solver" function to find itting parameters that best described the measured P-V curve. The best fit was defined as a curve resulting in the smallest root mean square between the measured and 
calculated P-V points. Minimizing the residual sum of squares (RSS) is a standard method employed for fitting curves. The mean fitting parameter of all study subjects was used to plot a mean P-V curve.

Abdominal compliance $\left(\mathrm{C}_{\mathrm{AB}}\right)$ was defined as a measure of the ease of abdominal expansion, expressed as a change in intra-abdominal volume (IAV) per change in intraabdominal pressure (IAP): $\mathrm{AC}=\Delta \mathrm{IAV} / \Delta \mathrm{IAP}[1] . \mathrm{C}_{\mathrm{AB}}$ is given as milliliter/centimeter of water for easier comparison with respiratory compliance.

Mann-Whitney rank sum test or Wilcoxon signed rank test was used as appropriate. A $p$ value of $<0.5$ was considered statistically significant.

\section{Results}

The subjects had a mean (SD) weight of $41.4(+/-8.5) \mathrm{kg}$ and end-expiratory lung volume of $1.68(0.30) \mathrm{L}$. Baseline IAP was $5.0 \mathrm{cmH}_{2} \mathrm{O}(3.7 \mathrm{mmHg})$. Expiratory tidal volume of 331 (68) $\mathrm{mL}$ and respiratory rate 34.1 (5.0) per minute were set at baseline (PEEP $5 \mathrm{cmH}_{2} \mathrm{O}$, no abdominal inflation). At $5 \mathrm{cmH}_{2} \mathrm{O}$ PEEP, the highest applied IAP ranged from 42.1 to $55.7 \mathrm{cmH}_{2} \mathrm{O}$ (31 to $41 \mathrm{mmHg}$ ), and additional IAV ranged from 7.7 to $14.3 \mathrm{~L}$.

In comparison with the Venegas equation, the alternate exponential equation produced a P-V curve that better fitted the measured values (lower root mean square) (Additional file 1: Figure S1-P-V curves using Venegas and exponential equation, Additional file 2: Table S1-Equation parameters of intra-abdominal and airway pressure-volume curves).

We therefore subsequently used the alternate exponential equation. Figure 1 depicts P-V curves derived from the exponential equation of the average and of each individual of these animals. To calculate an expected IAP from a given additional IAV, the alternate exponential equation $V=v+k \times \operatorname{Ln}\left(P_{0}-p\right)$ can be rearranged to $P_{0}=p+\operatorname{Exp}(V-v) / k$.

After maximal abdominal inflation was achieved using on average 10.4 L (2.1) additional IAV, IAP after initial abdominal inflation at $5 \mathrm{cmH}_{2} \mathrm{O}$ of positive endexpiratory pressure (PEEP) was higher than after subsequent abdominal re-inflation using the same additional IAV at $15 \mathrm{cmH}_{2} \mathrm{O}$ of PEEP, 49.4 (4.1) vs 45.6 (2.3) $\mathrm{cmH}_{2} \mathrm{O}$, respectively $(p=0.03)$ (Additional file 3: Figure S3-P-V curves at PEEP of 5 and $\left.15 \mathrm{cmH}_{2} \mathrm{O}\right)$. The abdominal compliance $\left(\mathrm{C}_{\mathrm{AB}}\right)$ at maximal IAV and IAP after initial

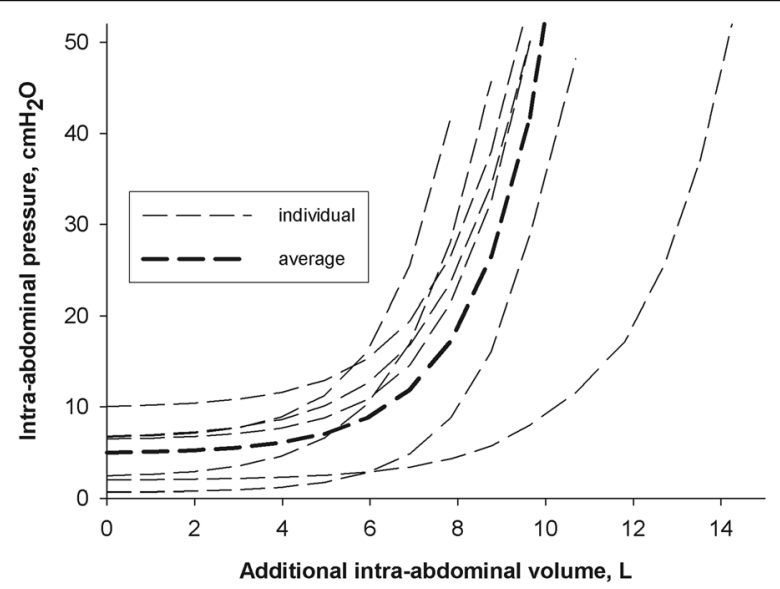

Fig. 1 Pressure volume curves showing intra-abdominal pressure (IAP) in centimeter of water in function of increasing additional intra-abdominal volume in liters. An exponential equation was used to calculate IAP for individual animals (narrow dashed curve) and for the average of all animals (bold dashed curve) 
(5 $\mathrm{cmH}_{2} \mathrm{O}$ PEEP) and repeat inflation $\left(15 \mathrm{cmH}_{2} \mathrm{O}\right.$ PEEP) were $55.3(5.0) \mathrm{mL} / \mathrm{cmH}_{2} \mathrm{O}$ and $62.0(6.9) \mathrm{mL} / \mathrm{cmH}_{2} \mathrm{O}$, respectively $(p=0.06)$.

With an increasing amount of additional IAV, IAP and to a lesser extent $\mathrm{pP}_{\mathrm{AW}}$ increased exponentially (Fig. 2). There was a directly proportional relationship between delta $\mathrm{pP}_{\mathrm{AW}}$ as a function of delta IAP, with a strong correlation (delta $\mathrm{pP}_{\mathrm{AW}}=0.14+0.43 \times$ delta IAP, $R^{2}=0.83, p<0.001$ ). Hence, abdomino-thoracic transmission approximated $40 \%$ (Fig. 3 ). With increasing IAP, $\mathrm{C}_{\mathrm{AB}}$ and $\mathrm{C}_{\mathrm{RS}}$ decreased (Fig. 4).

We calculated the effect of an additional IAV of $500 \mathrm{~mL}$ on $\mathrm{pP}_{\mathrm{AW}}$ and IAP (Table 1). The addition of $500 \mathrm{~mL}$ IAV increased $\mathrm{pP}_{\mathrm{AW}}$ and IAP to a greater extent at higher grades of IAH than at lower grades of IAH (i.e., reduced compliance at higher levels of IAH).

\section{Discussion}

The main findings in this animal model were that (a) raising IAV increased $\mathrm{pP}_{\mathrm{AW}}$ and IAP in an exponential manner, (b) raising IAV decreased $C_{A B}$ and $C_{R S}$, and (c) there was approximately a $40 \%$ transmission of IAP to $\mathrm{pP}_{\mathrm{AW}}$.

\section{IAV increased $\mathrm{pP}_{\mathrm{AW}}$ and IAP in an exponential manner}

We aimed to characterize how abdominal volumes influenced airway pressures and IAP. The IAV that produced an IAP $>40 \mathrm{cmH}_{2} \mathrm{O}(30 \mathrm{mmHg})$ varied between subjects. We therefore explored functions that could describe generic P-V curves of all the animals studied.

Functions have the advantage of allowing extrapolations even in the setting of a nonlinear curve if a certain number of pressure-volume $(\mathrm{P}-\mathrm{V})$ values are known. We first tested the Venegas equation for the additional IAV $-\mathrm{pP}_{\mathrm{AW}}$ and IAP relationship frequently used to describe a respiratory P-V curve [12]. The Venegas equation has been used to describe P-V curves other than respiratory [13]. We found that our alternative exponential function characterized the changes in $\mathrm{pP}_{\mathrm{AW}}$ and IAP with additional IAV more accurately than the Venegas equation. Exponential equations have been used to describe respiratory P-V curves [15].

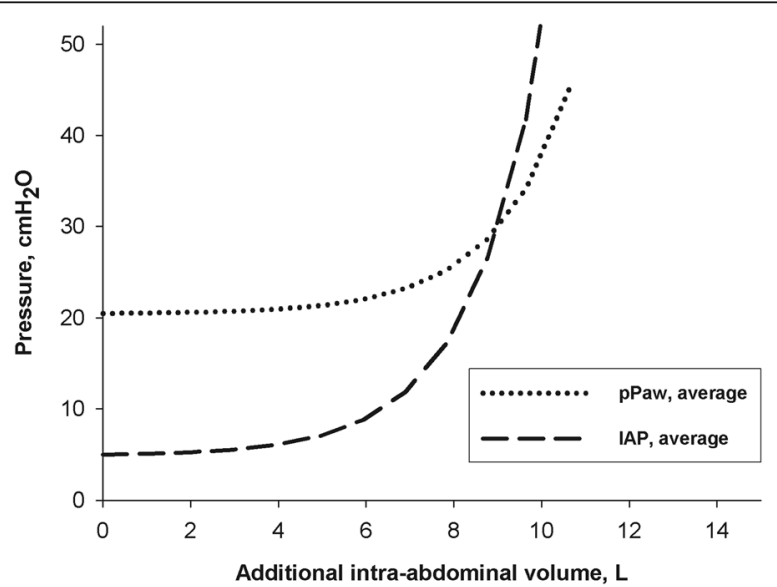

Fig. 2 Pressure volume curves showing intra-abdominal pressure (IAP) (dashed curve) and peak airway pressure $\left(\mathrm{PP}_{\mathrm{AW}}\right)$ (dotted curve) in centimeter of water in function of increasing additional intra-abdominal volume in liters. An exponential equation was used to calculate IAP and $\mathrm{PP}_{\mathrm{AW}}$ for the average of all animals 


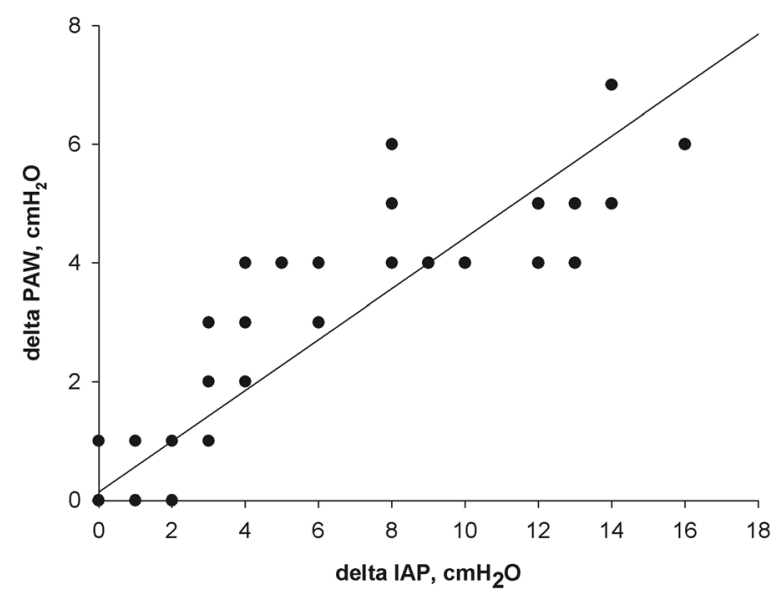

Fig. 3 Delta peak airway pressure $\left(\mathrm{PP}_{\mathrm{AW}}\right)$ in centimeter of water as a function of delta IAP in centimeter of water. Delta $\mathrm{pP}_{\mathrm{AW}}=0.14+0.43 \times$ delta IAP, $R^{2}=0.83, p<0.001$

An exponential relationship between IAV and IAP has been found in a previous animal study [16]. In a recent review article, we extracted all available human IAV and IAP measurements from the current literature [7]. In contrast to previous studies, we analyzed multiple IAV and IAP measurements that included multiple measurements and/or were derived from a larger IAP range (greater than $15 \mathrm{mmHg}$, the upper limit during laparoscopy). We found an exponential relationship between IAV and IAP. The pseudo-linear relationship between IAV and IAP found in previous studies can be explained by the relatively low IAP range and/or small number of measurements examined [4-6]. To our knowledge, we are the first to report an exponential relationship between IAV and $\mathrm{pP}_{\mathrm{AW}}$.

\section{Abdominal compliance}

After completion of the first P-V curves at $5 \mathrm{cmH}_{2} \mathrm{O}$ PEEP, we performed a second set of P-V curves at $15 \mathrm{cmH}_{2} \mathrm{O}$ PEEP levels. The higher $\mathrm{C}_{\mathrm{AB}}$ at $15 \mathrm{cmH}_{2} \mathrm{O}$ of PEEP indicates a

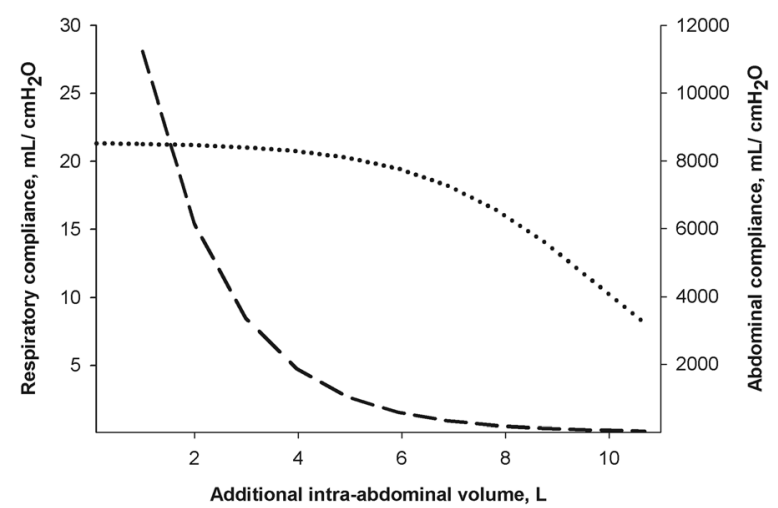

Fig. 4 Average abdominal compliance in milliliter per centimeter of water (dashed curve) and dynamic respiratory system compliance in milliliter per centimeter of water (dotted curve) as a function of increasing additional intra-abdominal volume in liters. Abdominal compliance was calculated from the difference of additional intra-abdominal volume per difference of resulting intra-abdominal pressure. Dynamic respiratory compliance was taken from the ventilator 


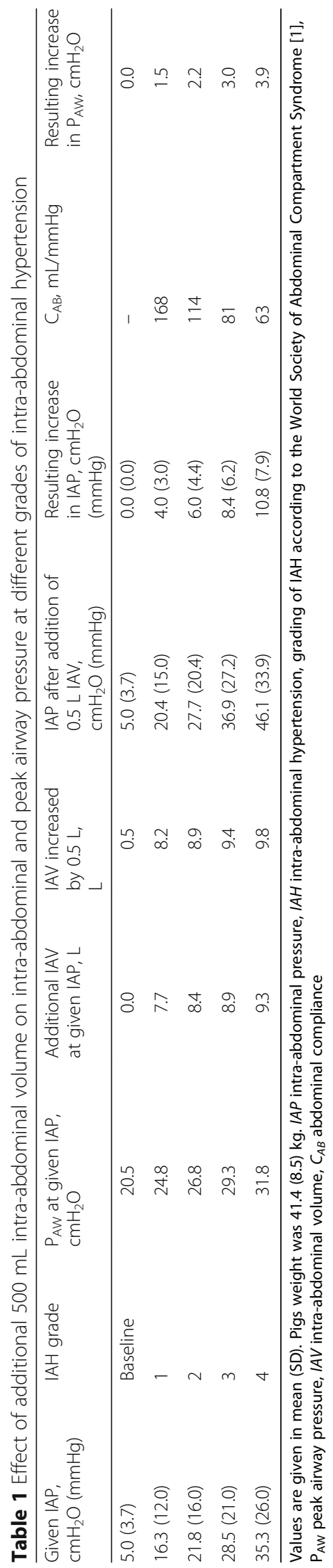


left shift of the P-V curves. This was opposite to what we anticipated and suggests that a considerable amount of "pre-stretching" occurred during the initial abdominal inflation rather than being a result of PEEP itself. We therefore only presented data obtained at PEEP of $5 \mathrm{cmH}_{2} \mathrm{O}$. Data from the literature suggests that stretching of the abdominal wall can lead to long-term changes in the elastic properties of the abdominal wall thereby improving abdominal compliance [7]. Unfortunately, we did not perform a third P-V curve at $5 \mathrm{cmH}_{2} \mathrm{O}$ PEEP following the P-V curve at $15 \mathrm{cmH}_{2} \mathrm{O}$ PEEP to confirm our hypothesis of the occurrence of pre-stretching.

Conceptually, three phases of abdominal pressure-volume behavior exist that occurs to some degree in parallel: the initial reshaping phase (minimal change in IAP despite large IAV change), the subsequent stretching phase, and finally, the pressurization phase (large IAP changes as a result of small IAV changes) [7]. Pre-stretching regularly occurs as an adaptive response to a chronic disease process (e.g., growing ascites or pregnancy), but it has also been shown to occur in the acute setting within a short period of time (e.g., during laparoscopy) [7].

The WSACS (www.wsacs.org) defines abdominal compliance as a measure of the ease of abdominal expansion, determined by the elasticity of the abdominal wall and diaphragm and expressed as a change in IAV per change in IAP $(\mathrm{L} / \mathrm{mmHg})[1,17,18]$. Not surprisingly additional IAV decreased $C_{A B}$ and $C_{R S}$.

\section{Abdomino-thoracic transmission}

We found approximately $40 \%$ of abdomino-thoracic transmission, whereby $\mathrm{pP}_{\mathrm{AW}}$ increased due to raising IAP. There is a paucity of literature examining the effect of various IAP on airway pressures. When averaging the results of three porcine studies, we found an approximate $40 \%$ abdomino-thoracic transmission [11, 19, 20]. In keeping with this, Cortes-Puentes and colleges found an approximate 50\% abdomino-thoracic transmission in pigs [21]. These results should be treated with caution as abdominothoracic transmission is likely to be different in critically ill patients. Factors such as obesity, presence of pleural effusions, and lung compliance may well substantially influence abdomino-thoracic transmission [22]. We could only locate one study in human subjects from which abdomino-thoracic transmission can be derived. Torquato et al. placed $5 \mathrm{~kg}$ weights on the abdomen of mechanically ventilated, critically ill patients. The average IAP increased from 10.5 to $15.6 \mathrm{cmH}_{2} \mathrm{O}$ and plateau airway pressures from 22.4 to $23.6 \mathrm{cmH}_{2} \mathrm{O}$ equating to an approximate $20 \%$ abdomino-thoracic transmission [23].

\section{Thoraco-abdominal transmission}

Thoraco-abdominal transmission can be explored by assessing the effect of either different PEEP levels or different tidal volumes on IAP. We attempted to examine the influence of PEEP on IAP. However, as we did not randomize the levels of PEEP and as described above, we believe that the unexpected findings may be the result of prestretching rather than the effect of different levels of PEEP. Therefore, we were unable to examine the influence of PEEP on thoraco-abdominal P-V curves.

Published reports suggest that PEEP has either no or minimal effect on IAP in animals and in humans [24]. We found in an animal experiment that PEEP did not influence IAP [8]. In humans, PEEP appears to increase IAP and the calculated 
thoraco-abdominal transmission ranges between 0.2 and $0.4 \mathrm{cmH}_{2} \mathrm{O}$ increase in IAP for each centimeter of water of PEEP [23, 25, 26]. Other studies have found tidal volume to have a significant impact on IAP [15].

The thoraco-abdominal transmission has been suggested to provide an estimate of the $\mathrm{C}_{\mathrm{AB}}$ by measuring the influence of different tidal volumes on the changes in IAP [18].

\section{How is this study useful?}

It is important for the critical care physician to be aware of the exponential nature of respiratory and abdominal pressure-IAV curve. An exponential pressure-volume relationship is the well-known Monro-Kellie doctrine applied in patients with intra-cranial hypertension [27].

At the lower IAV spectrum (i.e., patients with normal IAP), the respiratory and abdominal pressure-IAV curve is flat. This means that the abdominal cavity can accommodate several liters of additional IAV (i.e., intra- or retro-peritoneal hemorrhage) with little effect on airway or abdominal pressures. In using our pigs of around $40 \mathrm{~kg}$ as examples, an additional IAV of $4 \mathrm{~L}$ increased IAP by only $0.7 \mathrm{cmH}_{2} \mathrm{O}(0.5 \mathrm{mmHg})$ and the effect on airway pressures was negligible. Therefore, an absent rise in IAP does not exclude an intraabdominal or retroperitoneal hemorrhage in the lower IAV spectrum.

In the high IAV spectrum (i.e., patients with IAH), the respiratory and abdominal pressure-IAV curve is steep. Small changes in IAV can significantly affect airway pressure and IAP. Therefore, it is important to measure IAP regularly in patients at risk of developing IAH. Especially in patients with impending ACS, a small increase in IAV can easily progress to an ACS.

There is a high incidence of IAH in patients with respiratory failure [28]. IAH contributes to morbidity and mortality in patients with acute respiratory distress syndrome (ARDS) $[15,25,29,30]$. Airway pressures are often high when ventilating patients with ARDS, and it has been suggested that plateau pressure should be limited to $30 \mathrm{cmH}_{2} \mathrm{O}$ [31]. These recommendations do not take IAP into account even though IAH has been shown to increase airway pressures in this current study and in previous animal and human studies [8,32]. At least in patients with ARDS, recent studies suggest it is more important to limit the driving airway pressure than it is to limit plateau airway pressure [33]. Of note is that a sudden rise in airway pressures may reflect an acute increase in IAV and should prompt an abdominal examination to exclude an intra-abdominal pathology.

When aiming to reduce airway pressures and/or IAP in patients with IAH/abdominal compartment syndrome, it is useful to understand that small reductions in IAV can significantly improve airway pressure and IAP. In example, in this study, at grade IV IAH, a $500-\mathrm{mL}$ reduction in IAV reduced $\mathrm{pP}_{\mathrm{AW}}$ by $4 \mathrm{cmH}_{2} \mathrm{O}$ and IAP by $11 \mathrm{cmH}_{2} \mathrm{O}(8 \mathrm{mmHg})$. This observation is similar to the applied Monro-Kellie principle where drainage of small amount of cerebral spinal fluid can significantly reduce intra-cranial pressure in patients with intra-cranial hypertension [27].

There are multiple methods of reducing IAV, and the best management depends largely on the etiology of IAH [1]. Apart from diuresis (e.g., furosemide), renal replacement therapy, and surgery (e.g., removal of hematoma or decompressive laparotomy), percutaneous drainage of peritoneal fluid has been shown to be equally effective in reducing IAP [34-36]. In a case series, Reed et al. present 12 patients in which 
percutaneous drains were inserted in patients with IAH [35]. In the patients with higher pre-drainage IAP of smaller amount fluid removal led to greater decreases in IAP than in patients with smaller pre-drainage IAP.

\section{Limitations}

This study has several limitations: (a) These findings have been obtained in an animal model, limiting the transfer of our results into clinical practice. (b) We used a healthy lung model but critically ill patients frequently have injured lungs with reduced lung compliance. (c) This model assessed the effect of increasing IAV but not that of decreasing abdominal wall compliance on $\mathrm{pP}_{\mathrm{AW}}$ and IAP. Although decreased abdominal wall compliance does occur, increased IAV is the more dominant process in critically ill patients [7]. The abdominal closure may have decreased abdominal wall compliance [7]. (d) In clinical practice IAH arises more often on the basis of excess in intraabdominal fluid than of an excess in intra-abdominal gas. We used air to increase IAV but corrected the additional IAV to account for the compressibility of gas under pressure using the Boyle's equation. Although we visually ensured even distribution of the abdominal balloon we cannot exclude potential asymmetrical IAP distribution. (e) We measured mean IAP and not end-expiratory IAP as recommended by the WSACS [1]. (f) We measured dynamic respiratory compliance and peak airway pressure and not plateau airway pressure and static respiratory compliance. We assume that the same principles apply for plateau pressure. In a previous animal experiment [19], we found that plateau pressure paralleled peak airway pressure (data not published). (g) We did not observe any spontaneous diaphragmatic activity. However, we cannot totally rule out diaphragmatic activity potentially influencing our results as we did not use neuromuscular blocking agents. (h) We did not perform a third P-V curve at $5 \mathrm{cmH}_{2} \mathrm{O}$ PEEP following the $\mathrm{P}-\mathrm{V}$ curve at $15 \mathrm{cmH}_{2} \mathrm{O}$ PEEP to confirm our hypothesis that prestretching had occurred.

\section{Conclusions}

In conclusion, in an animal model, we found that raising IAV increased $\mathrm{pP}_{\mathrm{AW}}$ and IAP in an exponential manner. The exponential nature of IAV on $\mathrm{pP}_{\mathrm{AW}}$ and IAP suggests that the effect of a given change in IAV on $\mathrm{pP}_{\mathrm{AW}}$ and IAP will be greater at high than at low levels of IAP. In other words, in subjects with normal IAP, large increases in IAV will not affect airway pressure or IAP. But at high grades of IAH, small reductions in IAV can significantly improve airway and abdominal pressures.

\section{Additional files}

Additional file 1: Figure S1. Intra-abdominal pressure (IAP) in centimeter of water in function of increasing additional intra-abdominal volume (IAV) in liters. Example of one pig showing measured IAP values (crosses), calculated IAP values using Venegas equation (dotted curve) and exponential equation (dashed curve). Venegas equation: $V=a+\left[b /\left(1+e^{-(P-c) / d}\right)\right][12], V$ represents additional IAV, P represents absolute IAP, and $a, b, c$, and $d$ represents fitting parameters. Exponential equation: $V=V+k \times \operatorname{Ln}(P-p)$ where $V$ represents additional IAV, $P$ represents absolute IAP, and $v, k, p$ represents fitting parameters. (TIF $384 \mathrm{~kb}$ )

Additional file 2: Table S1. Equation parameters of intra-abdominal and airway pressure-volume curves. (DOCX 55 kb)

Additional file 3: Figure S3. Pressure-volume curves showing intra-abdominal pressure in centimeter of water at the initial PEEP level of $5 \mathrm{cmH}_{2} \mathrm{O}$ (dashed curve) and the subsequent PEEP level of $15 \mathrm{cmH}_{2} \mathrm{O}$ (solid curve) in function of increasing additional intra-abdominal volume in liters. (TIF $445 \mathrm{~kb}$ ) 


\section{Abbreviations}

$C_{A B}$ : Abdominal compliance; $C_{R S}$ : Dynamic respiratory system compliance; IAH: Intra-abdominal hypertension; IAP: Intra-abdominal pressure; IAV: Intra-abdominal volume; PEEP: Positive end-expiratory pressure; PPAW: Peak airway pressure; P-V: Pressure-volume

\section{Acknowledgements}

The work was performed at the Large Animal Facility of the University of Western Australia, Perth, Australia. Bench work and data analysis were carried out at the Sir Charles Gairdner Hospital.

We thank the Department of Medical Technology and Physics at the Sir Charles Gairdner Hospital as well as the team of the Large Animal Facility of the University of Western Australia and Brigit Roberts from the Intensive Care Unit of the Sir Charles Gardner Hospital for the technical assistance.

\section{Funding}

This study was supported by the Sir Charles Gairdner Hospital Research Fund (No 0810) and by local research funds of the Intensive Care Unit at the Sir Charles Gairdner Hospital.

\section{Availability of data and materials}

The datasets used and/or analyzed during the current study available from the corresponding author on a reasonable request.

\section{Authors' contributions}

AR, BN, and PVH participated in the conception, hypothesis delineation, and design of the study. AR and LH contributed to data acquisition. AR and BN performed data and statistical analyses. AR drafted the manuscript. BDK, BS, and PVH performed the first revision. All authors read and approved the final manuscript.

\section{Competing interests}

The authors declare that they have no competing interests.

\section{Ethics approval}

The Animal Ethics Committee of the University of Western Australia approved the study protocol (UWA RA/3/100/688). The study conformed to the regulations of the Australian code of practice for the care and use of animals for scientific purposes.

\section{Author details}

IIntensive Care Unit, Fiona Stanley Hospital, 102-118 Murdoch Drive, Murdoch (Perth), WA 6150, Australia. ${ }^{2}$ Intensive Care Unit, Sir Charles Gairdner Hospital, Hospital Avenue, Nedlands (Perth), WA 6009, Australia. ${ }^{3}$ School of Medicine and Pharmacology, The University of Western Australia, Sterling Highway, Crawley (Perth), WA 6009, Australia. ${ }^{4}$ Medical School, The Notre Dame University, Henry Road, Fremantle (Perth), WA 6959, Australia. ${ }^{5}$ Department of Anaesthesia and Perioperative Medicine, The Alfred Hospital, Commercial Road, Prahran (Melbourne), VIC 3181, Australia. ${ }^{6}$ Department of Pulmonary Physiology and Sleep Medicine, Sir Charles Gairdner Hospital, Hospital Avenue, Nedlands (Perth), WA 6009, Australia. ${ }^{7}$ Faculty of Science, The University of Western Australia, Sterling Highway, Crawley (Perth), WA 6009, Australia. ${ }^{8}$ West Australian Sleep Disorders Research Institute, QE II Medical Centre, Nedlands (Perth), WA 6009, Australia. ${ }^{9}$ General Intensive Care Unit, Hadassah University Hospital, Kiryat Hadassah, Jerusalem 91120, Israel.

Received: 6 December 2016 Accepted: 17 February 2017

Published online: 28 February 2017

\section{References}

1. Kirkpatrick AW, Roberts DJ, De Waele J et al (2013) Intra-abdominal hypertension and the abdominal compartment syndrome: updated consensus definitions and clinical practice guidelines from the World Society of the Abdominal Compartment Syndrome. Intensive Care Med 39:1190-1206

2. Malbrain ML, Chiumello D, Pelosi P et al (2004) Prevalence of intra-abdominal hypertension in critically ill patients: a multicentre epidemiological study. Intensive Care Med 30:822-829

3. Malbrain ML, Chiumello D, Pelosi P et al (2005) Incidence and prognosis of intraabdominal hypertension in a mixed population of critically ill patients: a multiple-center epidemiological study. Crit Care Med 33:315-322

4. Dejardin A, Robert A, Goffin E (2007) Intraperitoneal pressure in PD patients: relationship to intraperitoneal volume, body size and PD-related complications. Nephrol Dial Transplant 22:1437-1444

5. Papavramidis TS, Michalopoulos NA, Mistriotis G, Pliakos IG, Kesisoglou II, Papavramidis ST (2011) Abdominal compliance, linearity between abdominal pressure and ascitic fluid volume. J Emerg Trauma Shock 4:194-197

6. Fischbach M, Terzic J, Gaugler C et al (1998) Impact of increased intraperitoneal fill volume on tolerance and dialysis effectiveness in children. Adv Perit Dial 14:258-264

7. Blaser AR, Bjorck M, De Keulenaer B, Regli A (2015) Abdominal compliance: a bench-to-bedside review. J Trauma Acute Care Surg 78:1044-1053

8. Regli A, Hockings LE, Musk GC et al (2009) The Impact of positive end-expiratory pressure at different intra-abdominal pressures on functional residual capacity and oxygen delivery in a pig model. Intensive Care Med 35:S227, 0878

9. De Keulenaer B, Regli A, De Laet I, Roberts D, Malbrain ML (2015) What's new in medical management strategies for raised intra-abdominal pressure: evacuating intra-abdominal contents, improving abdominal wall compliance, pharmacotherapy, and continuous negative extra-abdominal pressure. Anaesthesiol Intensive Ther 47:54-62

10. Regli A, De Keulenaer B, De Laet I, Roberts D, Dabrowski W, Malbrain ML (2015) Fluid therapy and perfusional considerations during resuscitation in critically ill patients with intra-abdominal hypertension. Anaesthesiol Intensive Ther 47:45-53 
11. Regli A, Hockings LE, Musk GC et al (2010) Commonly applied positive end-expiratory pressures do not prevent functional residual capacity decline in the setting of intra-abdominal hypertension: a pig model. Crit Care 14:R128

12. Venegas JG, Harris RS, Simon BA (1998) A comprehensive equation for the pulmonary pressure-volume curve. J Appl Physiol 84:389-395

13. Williamson JP, McLaughlin RA, Noffsinger WJ et al (2011) Elastic properties of the central airways in obstructive lung diseases measured using anatomical optical coherence tomography. Am J Respir Crit Care Med 183:612-619

14. Colebatch HJ, Ng CK, Nikov N (1979) Use of an exponential function for elastic recoil. J Appl Physiol Respir Environ Exerc Physiol 46:387-393

15. Ranieri VM, Brienza N, Santostasi S et al (1997) Impairment of lung and chest wall mechanics in patients with acute respiratory distress syndrome: role of abdominal distension. Am J Respir Crit Care Med 156:1082-1091

16. Yoshino O, Quail A, Oldmeadow C, Balogh ZJ (2012) The interpretation of intra-abdominal pressures from animal models: the rabbit to human example. Injury 43:169-173

17. Malbrain ML, Roberts DJ, De Laet I et al (2014) The role of abdominal compliance, the neglected parameter in critically ill patients - a consensus review of 16. Part 1: definitions and pathophysiology. Anaesthesiol Intensive Ther 46:392-405

18. Malbrain ML, De Laet I, De Waele JJ et al (2014) The role of abdominal compliance, the neglected parameter in critically ill patients-a consensus review of 16. Part 2: measurement techniques and management recommendations. Anaesthesiol Intensive Ther 46:406-432

19. Regli A, Chakera J, De Keulenaer BL et al (2012) Matching positive end-expiratory pressure to intra-abdominal pressure prevents end-expiratory lung volume decline in a pig model of intra-abdominal hypertension. Crit Care Med 40:1879-1886

20. Regli A, Mahendran R, Fysh ET et al (2012) Matching positive end-expiratory pressure to intra-abdominal pressure improves oxygenation in a porcine sick lung model of intra-abdominal hypertension. Crit Care 16:R208

21. Cortes-Puentes GA, Cortes-Puentes LA, Adams AB, Anderson CP, Marini JJ, Dries DJ (2013) Experimental intraabdominal hypertension influences airway pressure limits for lung protective mechanical ventilation. J Trauma Acute Care Surg 74:1468-1473

22. O'Quin RJ, Marini JJ, Culver BH, Butler J (1985) Transmission of airway pressure to pleural space during lung edema and chest wall restriction. J Appl Physiol (1985) 59:1171-1177

23. Torquato JA, Lucato JJ, Antunes T, Barbas CV (2009) Interaction between intra-abdominal pressure and positiveend expiratory pressure. Clinics (Sao Paulo) 64:105-112

24. De Keulenaer BL, De Waele JJ, Powell B, Malbrain ML (2009) What is normal intra-abdominal pressure and how is it affected by positioning, body mass and positive end-expiratory pressure? Intensive Care Med 35:969-976

25. Krebs J, Pelosi P, Tsagogiorgas C, Alb M, Luecke T (2009) Effects of positive end-expiratory pressure on respiratory function and hemodynamics in patients with acute respiratory failure with and without intra-abdominal hypertension: a pilot study. Crit Care 13:R160

26. Verzilli D, Constantin JM, Sebbane M et al (2010) Positive end-expiratory pressure affects the value of intra-abdominal pressure in acute lung injury/acute respiratory distress syndrome patients: a pilot study. Crit Care 14:R137

27. Wilson MH (2016) Monro-Kellie 2.0: The dynamic vascular and venous pathophysiological components of intracranial pressure. J Cereb Blood Flow Metab 36:1338-1350

28. Holodinsky JK, Roberts DJ, Ball CG et al (2013) Risk factors for intra-abdominal hypertension and abdominal compartment syndrome among adult intensive care unit patients: a systematic review and meta-analysis. Crit Care 17:R249

29. Gattinoni L, Pelosi P, Suter PM, Pedoto A, Vercesi P, Lissoni A (1998) Acute respiratory distress syndrome caused by pulmonary and extrapulmonary disease. Different syndromes? Am J Respir Crit Care Med 158:3-11

30. Rouby JJ, Puybasset L, Nieszkowska A, Lu Q (2003) Acute respiratory distress syndrome: lessons from computed tomography of the whole lung. Crit Care Med 31:S285-S295

31. Marini JJ, Gattinoni L (2004) Ventilatory management of acute respiratory distress syndrome: a consensus of two. Crit Care Med 32:250-255

32. Hedenstierna G, Larsson A (2012) Influence of abdominal pressure on respiratory and abdominal organ function Curr Opin Crit Care 18:80-85

33. Laffey JG, Bellani G, Pham T et al (2016) Potentially modifiable factors contributing to outcome from acute respiratory distress syndrome: the LUNG SAFE study. Intensive Care Med 42:1865-1876.

34. Sun ZX, Huang HR, Zhou H (2006) Indwelling catheter and conservative measures in the treatment of abdominal compartment syndrome in fulminant acute pancreatitis. World J Gastroenterol 12:5068-5070

35. Reed SF, Britt RC, Collins J, Weireter L, Cole F, Britt LD (2006) Aggressive surveillance and early catheter-directed therapy in the management of intra-abdominal hypertension. J Trauma 61:1359-1363, discussion 1363

36. Cheatham ML, Safcsak K (2011) Percutaneous catheter decompression in the treatment of elevated intraabdominal pressure. Chest 140:1428-1435 